\title{
Breast Cancer Detection Using Automated Breast Ultrasound in Mammographically Dense Breasts
}

\author{
SHERINE K. AMIN, M.D.; MOHAMED G. ABDEL MUTALEB, M.D. and LATIFA E. GAD, M.Sc.
}

The Department of Radiodiagnosis, Faculty of Medicine, Ain Shams University

\begin{abstract}
Background: Automated Breast Ultrasound technology (ABUS) allows the radiologist to interpret ultrasonography images in a separate time after acquisition. Different interpretation times have been reported, ranging from 5 to $10 \mathrm{~min}$, probably according to differences in readers' experience and complexity of each case.

Aim of Study: To detect the impact of ABUS technique's advantages, pearls and pitfalls combining with mammography compared with mammography alone, significantly improved detection of breast cancers in women with dense breast tissue without substantially affecting specificity.

Patients and Methods: This cross-sectional study was conducted on of 20 women at Radiodiagnosis Department, Shoubra General Hospital referred from surgery clinic during a period of about one year. The study was limited to only females who were willingness to undergo additional investigations after being diagnosed as dense breast on mammography.

Results: We found that cases with ABUS study shows sensitivity about $(60 \%)$ which is more than that of mammogram $(30 \%)$ but less than HHUS (80\%); while ABUS (70\%) was less specific than both mammogram (100\%) and HHUS (90\%). Accuracy of HHUS (85\%) was more than that of both mammogram (65\%) and ABUS (65\%), with $p$-value (0.257) to both mammogram and ABUS, and (0.008) to HHUS.

Conclusion: Adding automated breast ultrasound to mammography is of great value in detection of breast cancer in mammographically dense breasts. It increases the detection rate of breast lesions mostly cancer. It is important as screening tool to decrease doses of radiation that female exposed to while mammogram screening.
\end{abstract}

Key Words: Breast cancer - Automated breast ultrasoundDense breasts.

\section{Introduction}

BREAST cancer is the most commonly diagnosed malignancy in women worldwide and is the second leading cause of cancer death in women in the

Correspondence to: Dr. Sherine K. Amin, The Department of Radiodiagnosis, Faculty of Medicine, Ain Shams University
United States. Early detection of breast cancer improves outcomes. Screening strategies for detecting early stage breast cancer are now stratified [1].

Mammography has been proven in randomized controlled trials to be a sensitive screening tool for the detection of early breast cancer. The reported sensitivity of screening mammography varies from $65 \%$ to $91 \%$ [2].

The performance of mammography is reduced for cancer detection in dense-breasted women as mammograms are summation images, with all breast tissue overlapping in each view. Cancers may not be visualized because of overlying dense breast tissue. Mammography can miss far posterior cancers in the retro-mammary space because of inadequate positioning of deep tissue [3]

Ultrasonography is currently considered the first-line examination in the detection and characterization of breast lesions including the evaluation of breast cancer. In spite of mammography consider as the primary method for screening especially the noteworthy ability of microcalcifications detection. US is good in mass or mass-like lesion detection, especially in the dense breast population [4]

Like traditional ultrasound, Automated Breast Ultrasound (ABUS) uses high-frequency sound waves targeted at the breast, but the scans provide physicians with a 3-D volumetric image of the entire breast. These 3-D images are more beneficial to women within the dense breast population because they give radiologists the ability to check the breast from a variety of angles and offer a better interpretation [5].

Automated Breast Ultrasound System is a comfortable, non-ionizing alternative to other supplemental screening options for women with dense 
breast tissue. When used in addition to mammography, ABUS can improve breast cancer detection by 55 percent over mammography alone [6] .

\section{Patients and Methods}

A cross-sectional study was performed at Radiodiagnosis Department, Shoubra General Hospital. The study population consisted of 20 women of mean age (35 to 55 years) referred from surgery clinic during a period of about one year (from November 2018 to January 2020). The study was limited to only females who were willing and give consent to undergo an additional investigations. aphy.

Inclusion criteria: Dense breast on mammog-

Exclusion criteria: Patients with fatty breasts.

The clinical presentation differ among the cases, most of them presented with palpable masses (variable size), some complained of pain, mass with axillary lymph nodes and one case presented with bloody discharge.

All patients were subjected to: Full history: Including positive family history, history of breast lesions and hormonal drug intake. Also any previous imaging investigation if present should be considered. Reassurance and explanation of the steps of the study in details to patients. Informed consents were taken from all cases. Mammography study, automated breast ultrasound, hand held ultrasound were done in all cases. Histopathology was done to all cases as a gold standard to confirm diagnoses.

\section{Technique:}

Mammography was done with the (Fujifilm digital mammography system FDR MS-3500) using the small focal spot (nominal size, $0.09 \mathrm{~mm}$; measured size, $0.14 \mathrm{~mm}$ ) for all exposures. Patients were entered into the study only after completion of the mammography examination, which include: Contact cranio-caudal and medio-lateral oblique projection mammograms of both breasts, any additional films (magnification views) that are judged to be necessary. Mammograms were reviewed for the breast density, presence of masses with detailed analysis of its site, type, number, border, calcifications, and size. Then interpreted before selection for study. Breast sonography (Automated Breast Ultrasound) was done, after mammography examination, using the (Invenia ABUS system, GE healthcare) which consists of an automated scanner that produces serial $1.7-\mathrm{mm}$-thick high-resolution sonograms of both breasts, coupled with several efficient modes of image review. Examination of each breast with all sequences take about from 5 mins (about one minute for the sweeping of the probe per view). Patient lies in the supine position, with the ipsilateral arm above the head. A lotion or gel and disposable membrane were used to aid as acoustic coupling.

Each breast was examined in three different positions, Antero-Posterior (AP), Lateral (LAT) including the pectoral muscle and medial (MED).

After scanning all quadrants of both breasts using ABUS, hand-held US was performed in all the patients. Hand held ultrasound also done using (GE ultrasound koreo, Ltd), using a $7-11 \mathrm{MHz}$ linear transducer. The technique performed for HHUS was done after exposure of the breasts with the patient lying supine and her ipsilateral hand raised above the head. The ultrasound probe was oriented perpendicular to the chest wall. Radial scanning technique, in a clockwise fashion, using the nipple as a center point was followed. Scanning of each breast quadrant in the sagittal and transverse planes was also performed and the examination time took about $20 \mathrm{~min}$ for both breasts. Also scanning of axilla was performed using HHUS to detected lymph nodes, which is not available through ABUS scan.

Results of the breast history, physical examination, mammography examination, automated breast ultrasound, and hand held ultrasound were available.

Pathologic correlation was undertaken for all patients who had breast biopsy within about 7-15 days of study.

\section{Statistical analysis:}

Descriptive statistics were used in terms of frequencies (number of cases) and percentages when appropriate. Accuracy was represented using the terms sensitivity, specificity, positive predictive value, and negative predictive value. Analytic statistics using $t$-test and chi-square tests were used to compare the clinical and imaging findings obtained using pathological diagnosis as the gold standard of the detected masses.

\section{Results}

Of the 20 women included in our study, the mean patient age was 43.40 years (standard deviation, 6.19 years; range, 35-55 years) who underwent mammography, the both ABUS and HHUS followed by biopsy. 
The clinical presentation differ among the cases, most of them presented with palpable masses (variable size), some complained of pain, mass with axillary lymph nodes and one case presented with bloody discharge.

Among the 20 patients studied, 10 women were found to have pathologically confirmed breast cancers.

On comparing the findings of breast biopsy and mammogram, only 10 cases were found negative by biopsy while 17 cases reported negative by mammogram.

Regarding positive cases, 10 cases were found positive by biopsy, while only 3 cases were suspected by mammogram with $30 \%$ sensitivity, $100 \%$ specificity, 100\% PPV, 58.82\% NPV and 65\% accuracy (Table 3 ).

On comparing the findings of breast biopsy and HHUS, 10 cases found benign by biopsy, and 11 cases reported benign by HHUS.

Regarding malignant cases, 10 cases were found malignant by biopsy, and 9 cases were suspected by Hand Held Ultrasound (HHUS) with $80 \%$ sensitivity, $90 \%$ specificity, $88.89 \%$ PPV, $81.82 \%$ NPV and $85 \%$ accuracy (Table 4 ).

On comparing the findings of breast biopsy and ABUS, 10 cases were found benign by biopsy, and 11 were found benign by ABUS. Regarding malignant cases, 10 cases were found malignant by biopsy, and 9 cases were suspected by ABUS with $60 \%$ sensitivity, $70 \%$ specificity, $66.67 \%$ PPV, 63.64 NPV and 65\% accuracy (Table 5).

We found that cases with ABUS study shows sensitivity about $(60 \%)$ which is more than that of mammogram (30\%) but less than HHUS (80\%); while ABUS (70\%) was less specific than both mammogram (100\%) and HHUS (90\%). Accuracy of HHUS $(85 \%)$ more than that of both mammogram $(65 \%)$ and ABUS $(65 \%)$, with $p$-value $(0.257)$ to both mammogram and ABUS, and (0.008) to HHUS.

Table (1): Distribution of the studied cases according to age (years) $(n=20)$.

\begin{tabular}{lcc}
\hline Age (years) & No. & $\%$ \\
\hline$<40$ & 7 & 35.0 \\
$\geq 40$ & 13 & 65.0 \\
Min.-max. & \multicolumn{2}{c}{$35.0-55.0$} \\
Mean \pm SD. & \multicolumn{2}{c}{$43.40 \pm 6.19$} \\
Median & \multicolumn{2}{c}{42.50} \\
\hline
\end{tabular}

Table (2): Distribution of the studied cases according to clinical examination $(n=20)$.

\begin{tabular}{lll}
\hline Clinical examination & No. & $\%$ \\
\hline Mass & 14 & 70.0 \\
Pain & 3 & 15.0 \\
Mass + LNs & 2 & 10.0 \\
Bloody discharge & 1 & 5.0 \\
\hline
\end{tabular}

Table (3): Agreement (sensitivity, specificity and accuracy) for biopsy $(n=20)$.

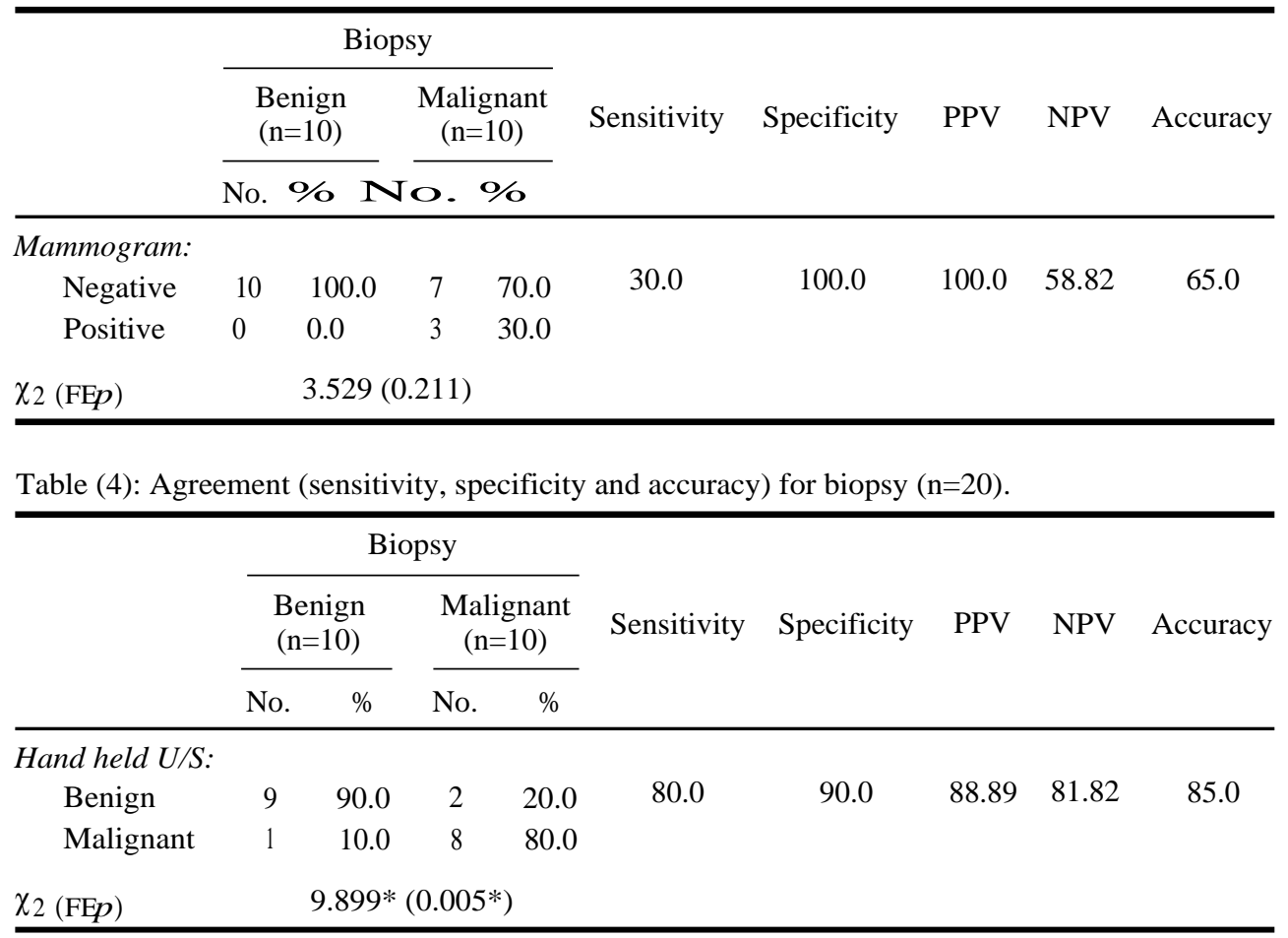


Table (5): Agreement (sensitivity, specificity and accuracy) for biopsy ( $\mathrm{n}=20)$.

\begin{tabular}{|c|c|c|c|c|c|c|c|c|c|}
\hline & \multicolumn{4}{|c|}{ Biopsy } & \multirow{3}{*}{ Sensitivity } & \multirow{3}{*}{ Specificity } & \multirow{3}{*}{ PPV } & \multirow{3}{*}{ NPV } & \multirow{3}{*}{ Accuracy } \\
\hline & \multicolumn{2}{|c|}{$\begin{array}{l}\text { Benign } \\
(\mathrm{n}=10)\end{array}$} & \multicolumn{2}{|c|}{$\begin{array}{l}\text { Malignant } \\
(\mathrm{n}=10)\end{array}$} & & & & & \\
\hline & No. & $\%$ & No. & $\%$ & & & & & \\
\hline \multicolumn{10}{|l|}{$A B U S:$} \\
\hline Benign & 7 & 70.0 & 4 & 40.0 & 60.0 & 70.0 & 66.67 & 63.64 & 65.0 \\
\hline Malignant & 3 & 30.0 & 6 & 60.0 & & & & & \\
\hline $\mathrm{X}^{2}\left({ }^{\mathrm{FE}} p\right)$ & \multicolumn{4}{|c|}{$1.818(0.370)$} & & & & & \\
\hline
\end{tabular}

Table (6): Comparison (sensitivity, specificity, accuracy and $p$-value) between Mammogram, HHUS and ABUS.

\begin{tabular}{lccl}
\hline & Mammogram & HHUS & ABUS \\
\hline Sensitivity & $30 \%$ & $80 \%$ & $60 \%$ \\
Specificity & $100 \%$ & $90 \%$ & $70 \%$ \\
Accuracy & $65 \%$ & $85 \%$ & $65 \%$ \\
$p$-value & 0.257 & 0.008 & 0.257 \\
\hline
\end{tabular}

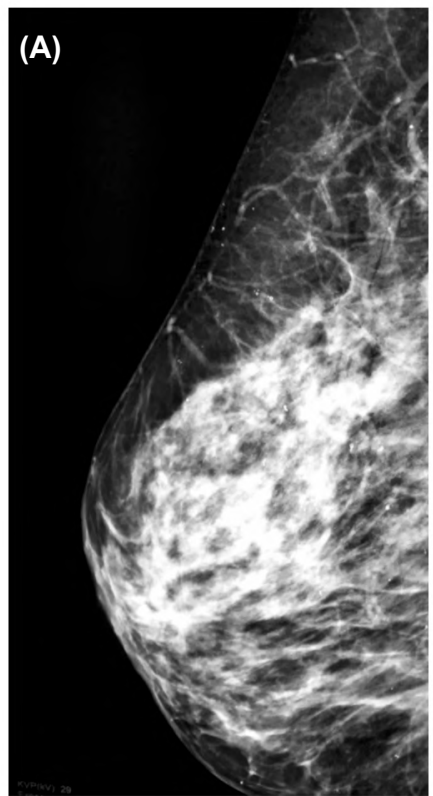

(C)

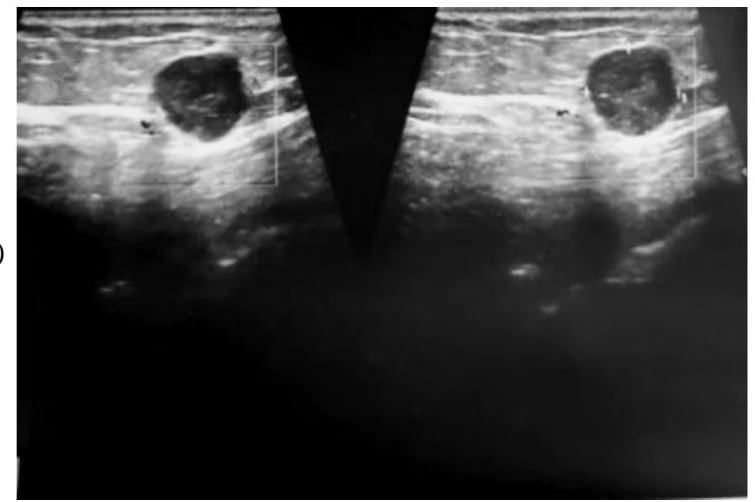

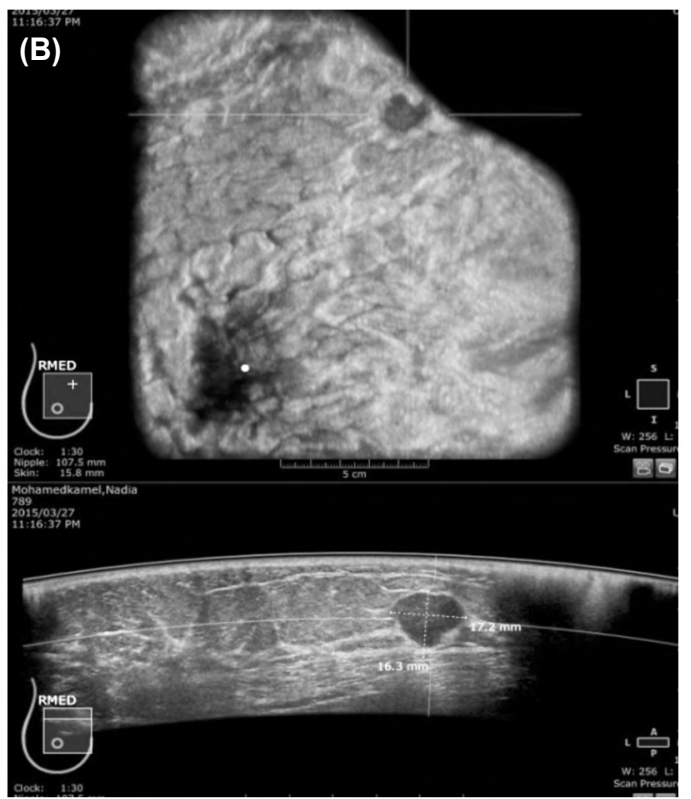

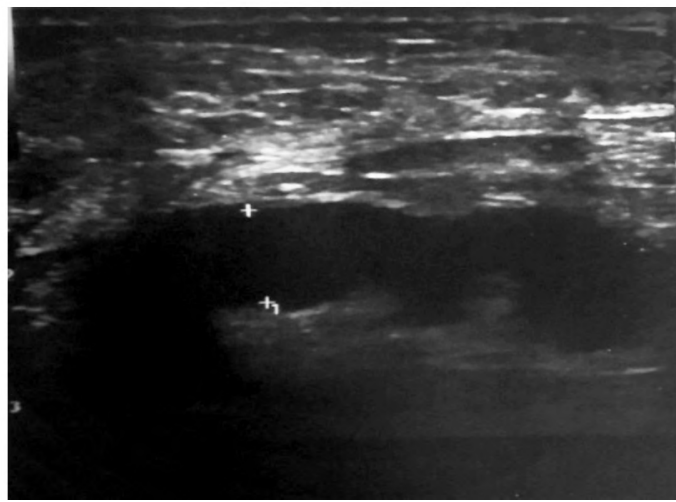

Fig. (1): A 39 year old female with right breast mass, (A) MLO view, ACR C right mammogram with tiny calcifications, (B) ABUS a: Coronal and b: Axial views show irregular hypoechoic lesion, (C) HHUS shows a: Round hypoechoic lesion with hyperechoic vascular lesion inside. b: Enlarged axillary lymph node with thick cortex. (Pathology: Ductal carcinoma insitu). 
Fig. (2): A 37 year old female with breast mass, (A): MLO view, ACR D right mammogram, (B): ABUS a: Co-ronal and b, axial views show right breast hypoechoic lesion with internal echoes, $(C)$ : HHUS shows well defined hypoechoic lesion with free mobile internal echoes (pathology: Complicated Cyst (cyst with debris).
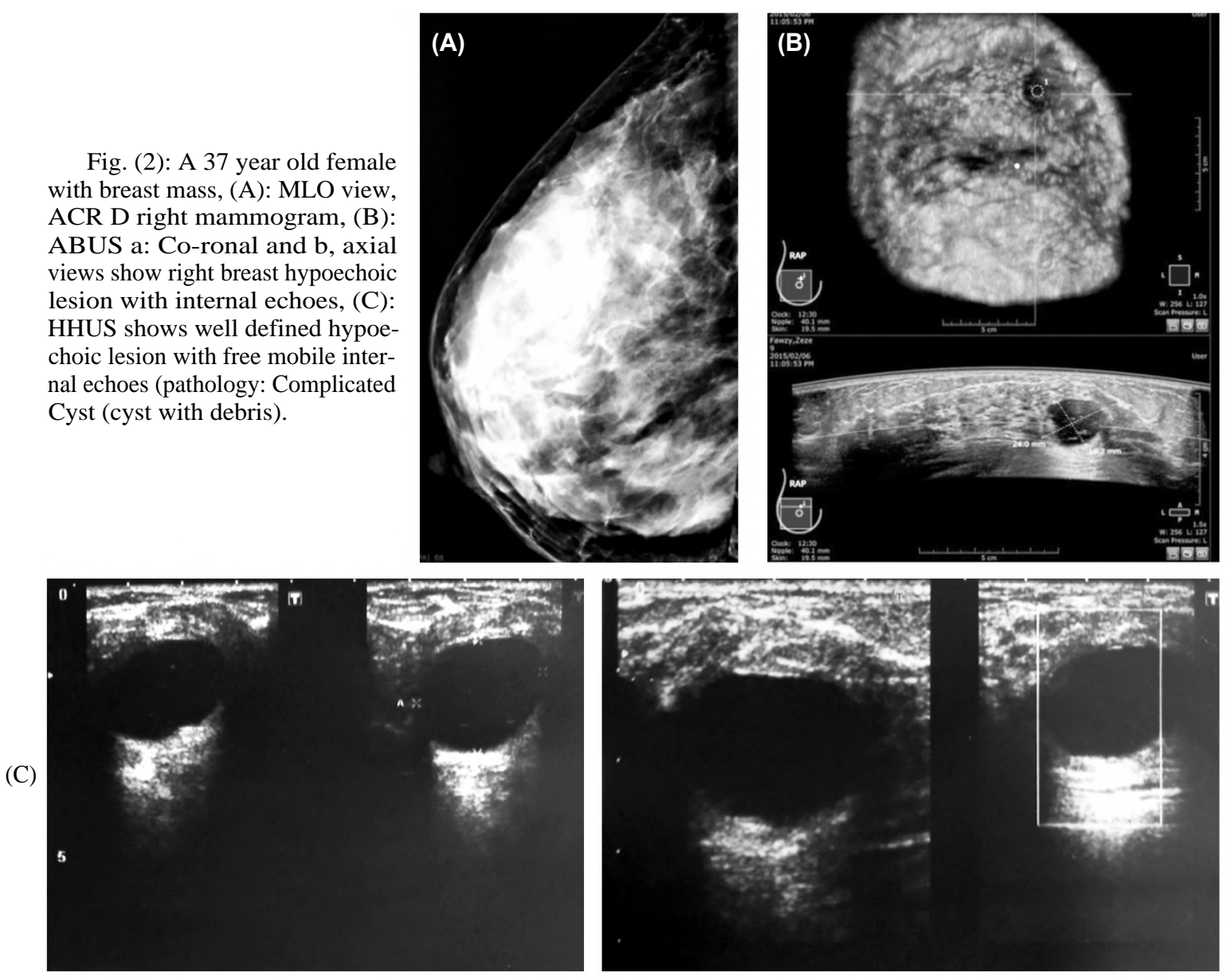

Fig. (3): A 40 year old female with right breast mass, (A): CC view, ACR $\mathrm{D}$ right mammogram, (B): ABUS a: Coronal and b: Axial views show ill defined lesion, (C): HHUS shows irregular hypoechoic lesion with multiple spiculations (pathology: Invasive ductal carcinoma).
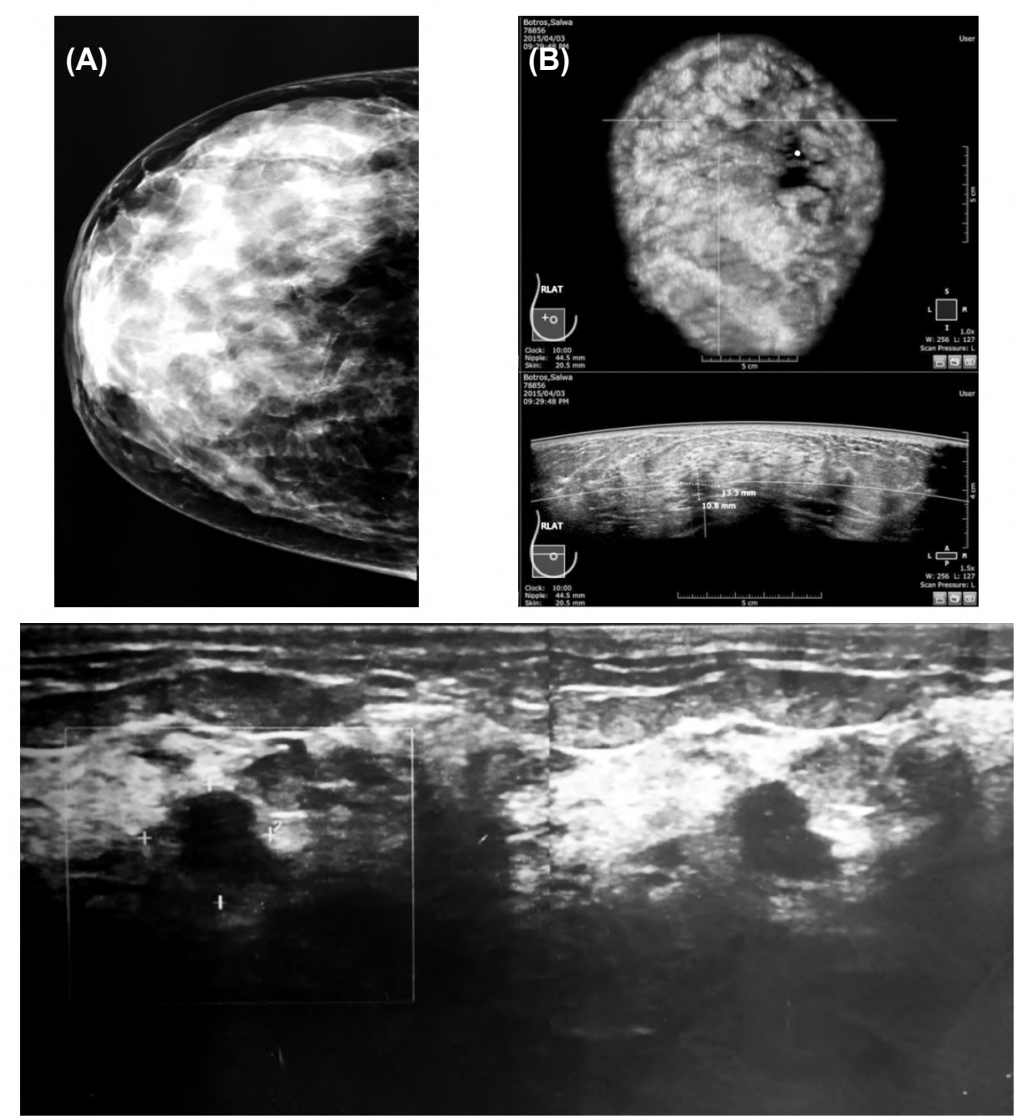


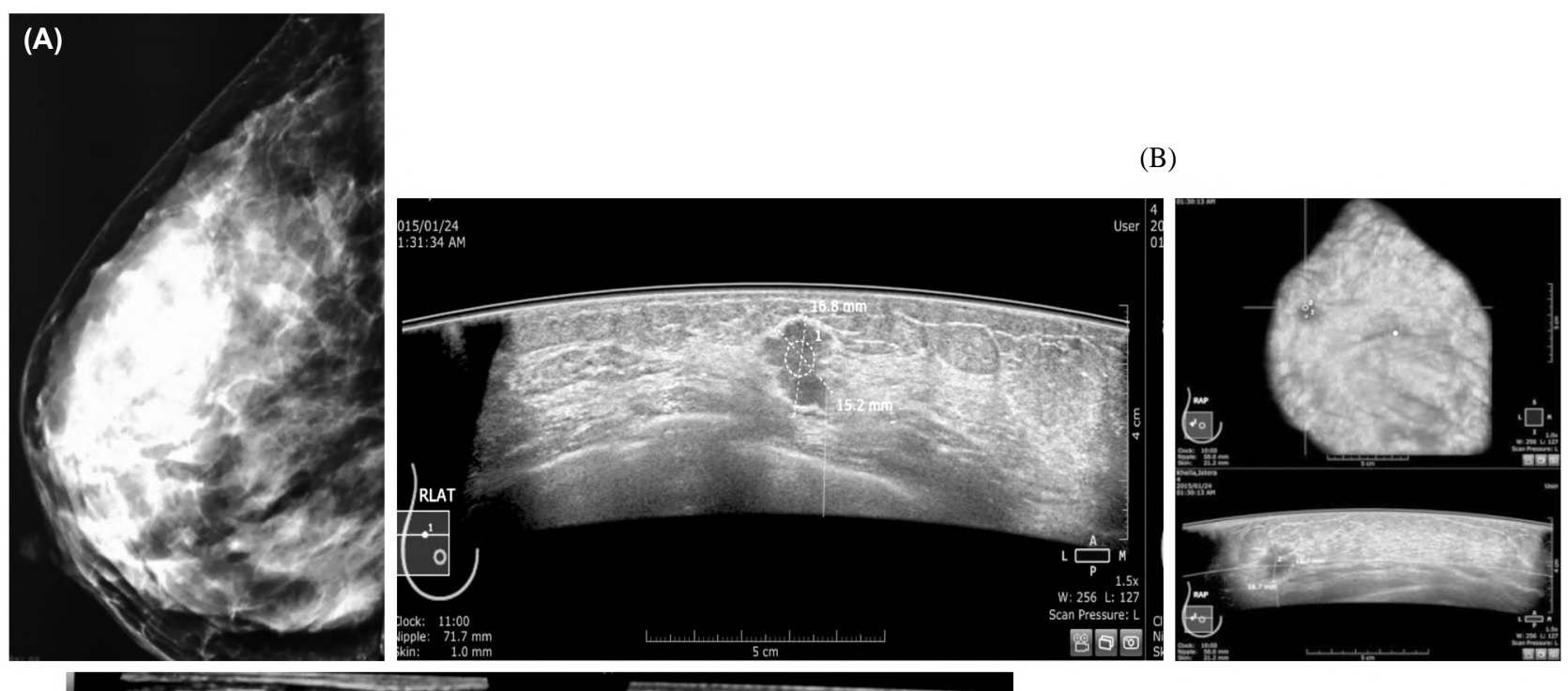

(C)
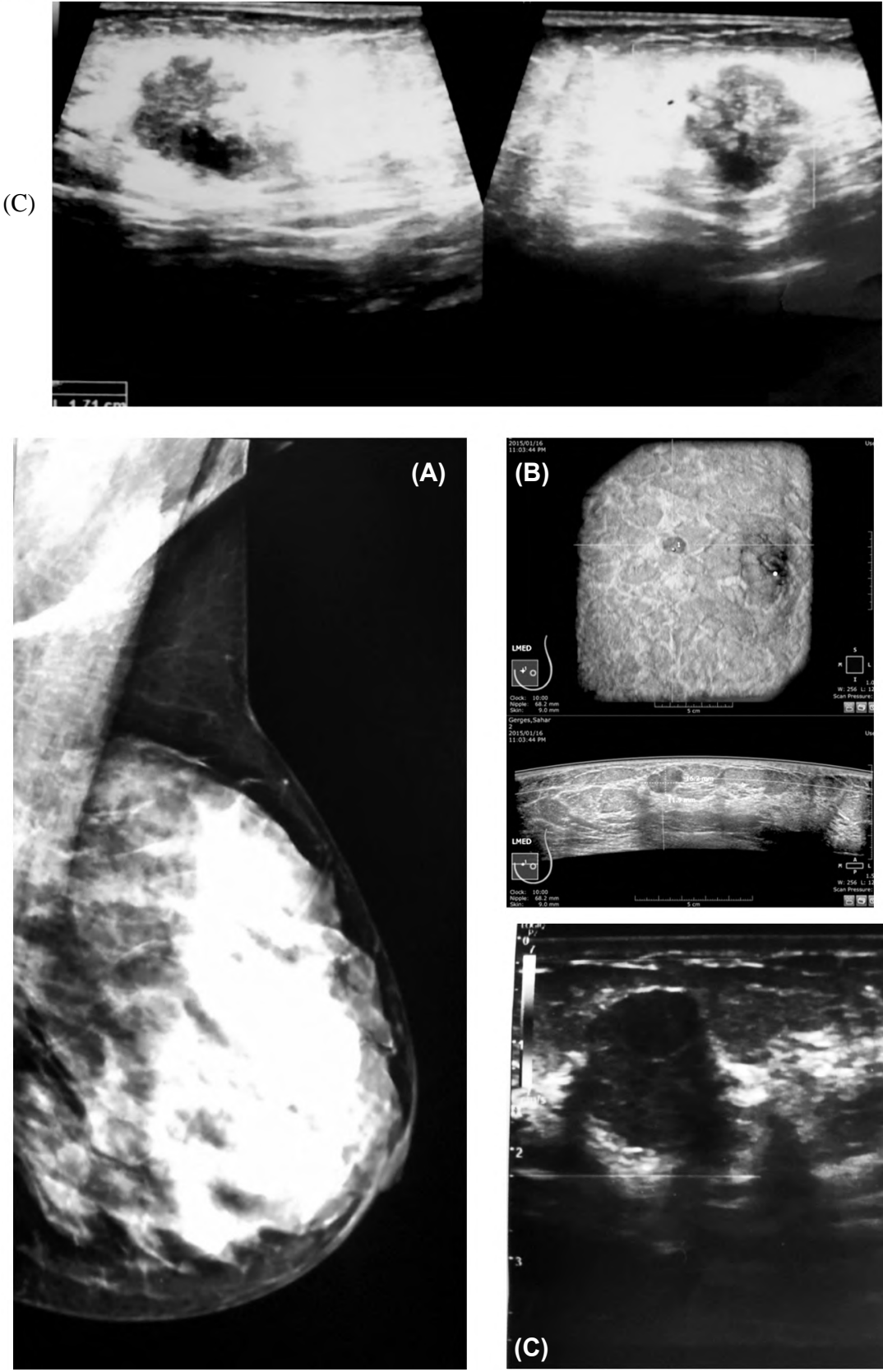

Fig. (4): A 50 year old with right breast mass, A) MLO view, ACR D right mammogram, B): ABUS a: Coronal and b: Axial views show irregular microlobulated lesion, C): HHUS shows irregular heterogeneous lesion with internal vascularity (Pathology: Mixed ductal and lobular carcinoma.
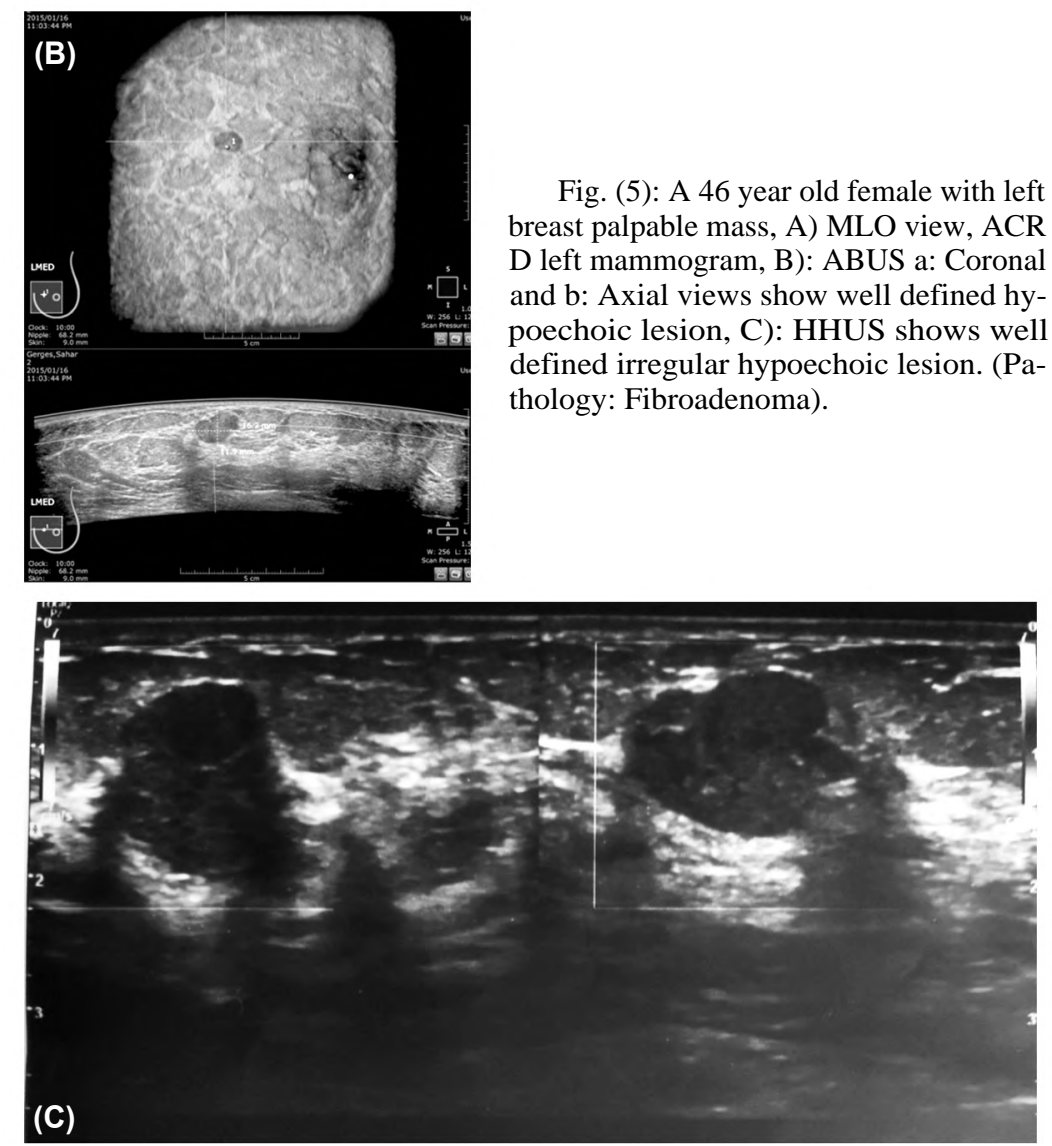


\section{Discussion}

Our results clearly show that it is feasible to implement ABUS into a high volume FFDSM center and increase the cancer detection rate in women aged $35-55$ years.

It shows that addition of ABUS screening in women with dense breast tissue at FFDSM demonstrated an increase in detection rate of cancers in women screened despite of false positive and false negative cases by ABUS. The improved incremental cancer detection rate was comparable to the rates observed in studies of mammography screening supplemented by HHUS in women with dense breasts. Adding HHUS has also been shown to increase the cancer detection rate in women of ages ranges from 35-55 year.

ABUS showing no considered (wide) difference at lesion description from HHUS except for color Doppler and Axillary lymph node scanning that not available in ABUS.

Janie et al., [7] reported that of 121 women with complete follow-up, twenty-four women (19.8\%) were recalled by at least one modality. Recalls increased from $5.0 \%$ to $13.2 \%(p=0.002)$ when ABUS was added to FFDM. Findings recalled by ABUS was more likely to result in a recommendation for short term follow-up imaging or tissue biopsy compared to findings recalled by only FFDM (100\% vs. $42.1 \%, p=0.041)$. The cancer detection rate was 8.3 per 1000 screens $(1 / 121)$.

The agreement between ABUS and HHUS examinations in the BI-RADS categorization of suspicious breast masses was good, results of Yun et al., [8] found that their study analyzed 135 breast lesions in 135 patients (median age, 49 years; range, 35 to 82 years) who underwent both ABUS and HHUS followed by biopsy. The pathological analysis revealed $49(36.3 \%)$ malignant lesions and $86(63.7 \%)$ benign lesions. The overall agreement between ABUS and HHUS in all cases was $79.3 \%$ (kappa $=0.61 ; p<0.001)$, while the agreement in confirmed malignancies was $55.1 \%$ (kappa $=0.39$, $p<0.001)$.

Unlike our study, Abd Elkhalek et al., [9] according their results of a study of twenty-five female patients, age ranging 29-69 years complaining from breast pain or a palpable mass were submitted to ABUS and mammography. In their study, ABUS system was applied on 25 patients of mean age 43.4 with standard deviation of \pm 9.08 . Eleven patients were below 40 years and 14 patients above 40 years. Thirteen patients (52\%) with dense breasts were ACR C and D (9 and 4, respectively), while 12 patients $(48 \%)$ were ACR A and B (4 and 8 , respectively). The percentage of BIRAD II and BIRAD III detected lesions in mammogram was $28 \%$ and $12 \%$, while, in ABUS, it increased to $32 \%$ and $20 \%$, respectively thus, ABUS is a useful tool in detection of benign lesions and probably benign lesions compared to mammogram. While in our study, we noticed no significant difference at BIRAD IV and V between the two studies. The sensitivity of the ABUS is about $100 \%$, and that means, in all the results of the mammogram study, ABUS can detect it without significant change, while the specificity of the ABUS was about $62 \%$ and was more evident in benign lesions. We found that the detected lesions by ABUS in the age group below 40 years was more than the mammogram (ABUS $=7$ cases and mammogram $=5$ cases), while in the age group above 40 years the ABUS found 1 case only more than the mammogram study.

Mostafa et al., [10] showed that there was a statistically difference between the number of patients with lesions detected by ABUS and HHUS with $p$-value of 0.012 . There was no statistically difference between ABUS and HHUS in the detection of multiple lesions in the same patients with $p$-value of 0.16 . Using mammography alone, lesions were detected in 24 out of 40 patients with positive findings, and addition of ABUS to mammography increased this number as lesions were detected in 38 out of 40 patients. A statistically significant difference was found with $p$-value $=0.0001$. The added value of ABUS to mammography in detection of breast lesions was most noted in patients with dense and extremely dense breasts (ACR C and D) as a statistically significant difference was found with $p$-value $=0.0001$. Using mammography alone, 20 out of 36 lesions were detected while with the addition of ABUS 34 out of 36 lesions were detected. A statistically significant difference was found between ABUS and HHUS in the detection of lesions smaller than $5 \mathrm{~mm}$, when compared to HHUS. The number of lesions detected by ABUS smaller than $5 \mathrm{~mm}$ was 10 compared to 4 by HHUS with a $p$-value of 0.002 .

Also Giger et al., [11] reported an increase in sensitivity from $40 \%$ for mammography alone to $81 \%$ with the addition of ABUS. In a recent study comparing FFDSM with a 3D ABUS to FFDSM alone for mammography negative cancers, the addition of 3D ABUS caused a $23.9 \%$ sensitivity increase.

Brem et al., [12] found that the relative increase in sensitivity for the 16 mammographic-negative 
cancers that did not have any prior breast interventions to be of special interest. This finding shows that the confidence and performance of screening interpretations are improved with the use of supplemental screening ABUS.

ABUS can provide additional information in the differential diagnosis of a lesion. It has significantly higher sensitivity than mammography, but it is similar to manual US and cannot be preferred to a manual US examination. As Wang et al., [13] reported that detection rate, diagnostic accuracy and mammography sensitivity were significantly lower than those of each US method $(p<0.05)$. There were no significant differences between manual US and ABUS. When combining ABUS, US and mammography, diagnostic accuracy, sensitivity and specificity reached $96.4 \%, 97.1 \%$ and $95.2 \%$, respectively. A spiculated and stellate margin in the coronal plane has a high specificity in diagnosing malignant lesions.

Another agreement to our study by Kelly et al., [3] as reported that breast cancer detections doubled from 23 to 46 in 6,425 studies using AWBU with mammography, resulting in an increase in diagnostic yield from 3.6 per 1,000 with mammography alone to 7.2 per 1,000 by adding AWBU (an additional 3.6 per 1,$000 ; 95 \% \mathrm{CI}=2.3-5.4)$. Sensitivity for mammography alone was $40 \%(95 \% \mathrm{CI}=27.5$ $54 \%$; 23 out of 57 cancers), but increased to $81 \%$ (95\% CI=68- 90\%; 46 out of 57 cancers) with the addition of AWBU. Sensitivity of AWBU alone was $67 \%$ (95\% CI=53-79\%; 38 out of 57$)$.

\section{Conclusion:}

According to our study, it shows that adding automated breast ultrasound to mammography is of great value in detection of breast cancer in mammographically dense breasts. It increases the detection rate of breast lesions mostly cancer. It is important as screening tool to decrease doses of radiation that female exposed to while mammogram screening.

But still hand held ultrasound is superior, it is of value in detection of breast lesions due to automated breast ultrasound pitfalls, and false positive and false negative cases.

\section{References}

1- RACHEL F.B., MEGAN J.L., JENNIFER L. and JESSICA T.: Screening Breast Ultrasound: Past, Present, and Future. A.J.R., 204: 234-40, 2015.

2- CRYSTAL P., STRANO S.D., SHCHARYNSKIAND S and KORETZ M.J.: Using Sonography to Screen Women with Mammographically Dense Breasts. A.J.R., 181: 17782, 2003.

3- KELLY K.M., DEAN J., COMULADA W.S. and LEE S.J.: Breast cancer detection using automated whole breast ultrasound and mammography in radiographically dense breasts. Eur. Radiol., 20: 734-42, 2010.

4- PAN H.B.: The Role of Breast Ultrasound in Early Cancer Detection. Journal of Medical Ultrasound, 24: 138-41, 2016.

5- NYANUE W.: ABUS The Future of Breast Screening. Imaging Technology news; p. 15-6, 2013.

6- BARCLAY A. and LANSDOWNE D.: Breast Imaging, Diagnostic imaging Europe, 8. 34: 35, 2016.

7- JANIE M., SAVANNAH C.L., GERALDINE J.P., DANIEL S.L., et al.: Double reading of automated breast ultrasound with digital mammography or digital breast tomosynthesis for breast cancer screening. Clinical Imaging, 55: 119-25, 2019.

8- YUN G., KIM S.M., YUN B.L., AHN H.S. and JANG M.: Reliability of automated versus handheld breast ultrasound examinations of suspicious breast masses. Ultrasonography, 38: 264-71, 2019.

9- ABD ELKHALEK Y., BASSIOUNY A.M. and HAMID R.W.: Automated breast ultrasound system (ABUS): Can it replace mammography as a screening tool? Egyptian Journal of Radiology and Nuclear Medicine, Dec. 1; 50 (1): 51, 2019.

10- MOSTAFA A.A., ELTOMEY M.A., ELAGGAN A.M and HASHISH A.A.: Automated breast ultrasound (ABUS) as a screening tool: Initial experience. Egyptian Journal of Radiology and Nuclear Medicine, 50: 37, 2019.

11- GIGER M.L., MARC F., EDWARDS A., PAPAIOANNOU J., JIANG Y. and BREM R.: Women's Imaging: Automated Breast Ultrasound in Breast Cancer Screening of Women With Dense Breasts: Reader Study of Mammography Negative and Mammography Positive Cancers. A.J.R., 206: 1341-50, 2016.

12- BREM R.F., TABÁR L., DUFFY S.W., et al.: Assessing improvement in detection of breast cancer with threedimensional automated breast US in women with dense breast tissue: The SomoInsight Study. Radiology, 274: 663-73, 2015.

13- WANG Z.L., HONG J., LI J.L., HUANG Y. and TANG J.: Comparison of automated breast volume scanning to hand-held ultrasound and mammography. La Radiologia Medica, 117: 1287-93, 2012. 


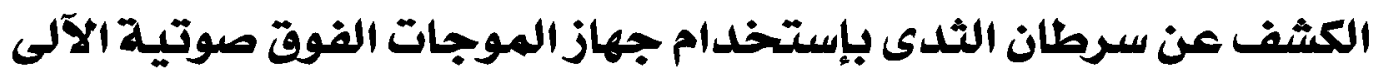 فى حالات الآثدية عالية عالية الكثافة بالماموجران}

المقدمة: تسمع تقنية تصوير الثدى بالموجات فهق الصوتية لآخصائى الآثعة بتفسير صود الموجات فهق الصوتية فى وقت منفصل بعد

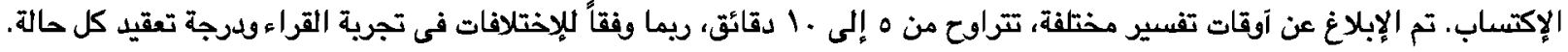

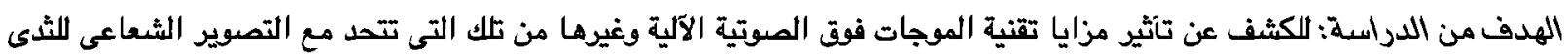

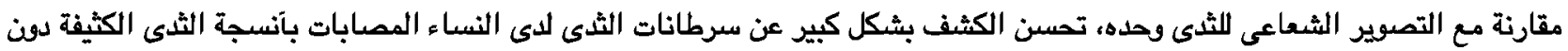
التآثير بشكل كبير على الخصوصية.

المرضى والطرق : آجريت هذه الدراسة المستعرضة على •r سيدة فى قسم التشخيص بالآثعة، مستشفى شبرا العام واللاتى تم تحويلهن

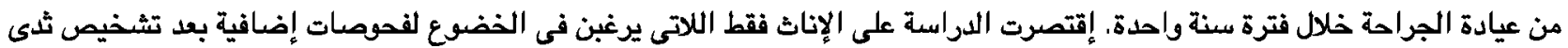
كثيف على التصوير الشعاعى اللثىى.

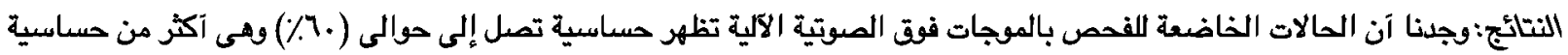

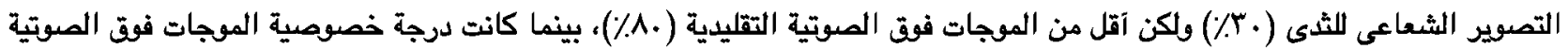

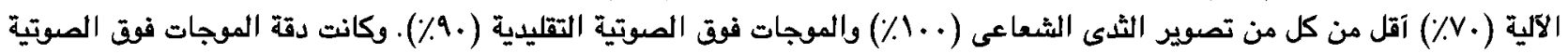

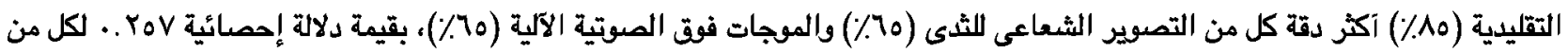

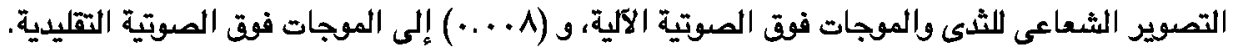

الخلاصة: إن لإضافة الموجات فوق الصوتية للثدى الآلى إلى التصرير الشعاعى للثدى قيمة كييرة فى الكثف عن سرطان الثىى فى الثَيين

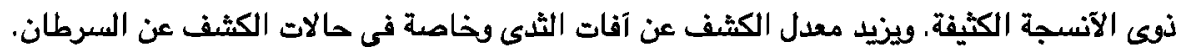

